\title{
Measuring the effect of electrostatic patch potentials in Casimir force experiments
}

\author{
Joseph L. Garrett $\odot,{ }^{1,2}$ Jongbum Kim $\oplus^{1,3}$ and Jeremy N. Munday $\circledast^{1,3}$ \\ ${ }^{1}$ Institute for Research in Electronics and Applied Physics, University of Maryland, College Park, Maryland 20742, USA \\ ${ }^{2}$ Department of Physics, University of Maryland, College Park, Maryland 20742, USA \\ ${ }^{3}$ Department of Electrical and Computer Engineering, University of California, Davis, California 95616, USA
}

(Received 1 March 2020; accepted 19 May 2020; published 17 June 2020; corrected 18 September 2020)

\begin{abstract}
The Casimir force is a consequence of quantum electrodynamic fluctuations, which induce interactions between materials. Patch potentials (i.e., spatial variations of electrostatic potentials across a surface) are a concern in measurements of the Casimir force because they can cause an additional force with a similar separation dependence. Previously, Kelvin probe force microscopy has been used to show that patch potentials on a flat surface cause an additional force that can reach over $1 \%$ of the value of the predicted Casimir force. Although nearly all Casimir force measurements are performed in a sphere-plate geometry, there has been little investigation into how the patches are distributed on the sphere. Here we present a measurement of the Casimir force between a sphere and a plate, where the electrostatic patch potentials are mapped on both surfaces and their effects are determined. Large patches are detected for gold deposited onto glass, but an ion-blocking layer is shown to reduce the voltage contrast and spatial extent of the patches. We find that the patch potential force is at least an order of magnitude less than the Casimir force when the sphere contains an ion-blocking layer; however, without this ion-blocking layer, the measured force can contain a significant electrostatic contribution, hence masking the Casimir force. Our results show the importance of measuring the electrostatic patches for individual Casimir force experiments.
\end{abstract}

DOI: 10.1103/PhysRevResearch.2.023355

\section{INTRODUCTION}

The electromagnetic fluctuations of the quantum vacuum lead to a force between metal surfaces, called the Casimir force [1]. In early measurements of the force, it was noted that spatial variations of the surface potential, called patch potentials, lead to a systematic overestimation of the force and such variations have continued to be a concern in subsequent measurements [2-10]. Patch potentials are also present in many sensitive experimental systems [11-13], and artifacts in other measurements have been attributed to patch potentials [14-16].

Variations of the electron chemical potential at the surface, from adsorbates or crystal orientation, lead to patch potentials on a conductor even when the bulk is at an equipotential $[17,18]$. Theoretical frameworks have been developed to determine the force caused by patch potentials [19-23], but their use is limited by the lack of accurate measurements of the potential on the interacting surfaces. Based on measurements of the potential on flat gold surfaces, the contribution of patch potentials has been estimated to be between 0.1 and $2 \%$ of the total force in the $<400-n m$ separation range [24-26], and other experiments have suggested the patch potential force can reach a magnitude comparable to the Casimir force

Published by the American Physical Society under the terms of the Creative Commons Attribution 4.0 International license. Further distribution of this work must maintain attribution to the author(s) and the published article's title, journal citation, and DOI. at several microns separation [7]. However, because most Casimir force measurements are performed in the sphere-plate geometry, measuring the potential on a plate does not suffice to completely characterize the residual electrostatic force.

In this article, we present a measurement of the spatial derivative of the Casimir force between two surfaces, a sphere and a plate, on which the patch potentials are quantitatively determined for each surface (Fig. 1). The method of de Man et al. [27-29] is used to measure the spatial derivative of the Casimir force. The potentials of the sphere and the plate are measured with Kelvin probe force microscopy (KPFM) $[26,30]$ before and after the force measurements. Although many techniques have been developed to measure the electric potential of surfaces [18,31,32], KPFM has the advantages of $<50$-nm spatial resolution, fast image acquisition rates, and environmental flexibility [33].

\section{IMAGING PATCH POTENTIALS}

KPFM imaging of patch potentials on the spherical probe is hindered by (i) the probe's small spring constant, which causes the probe to oscillate when scanned by typical frequency modulation KPFM, and (ii) the curvature of the sphere, which limits voltage sensitivity [34]. A heterodyne KPFM technique overcomes the first limitation by applying an $\mathrm{AC}$ voltage at a frequency far above the spherical probe's resonance frequency so that the probe is not excited, and it overcomes the second limitation by detecting all signals at the first resonance of the cantilever, so that the motion of the tip apex is nearly vertical and less affected by the curvature of the sphere [33,34]. KPFM is performed with Pt-coated Si probes 

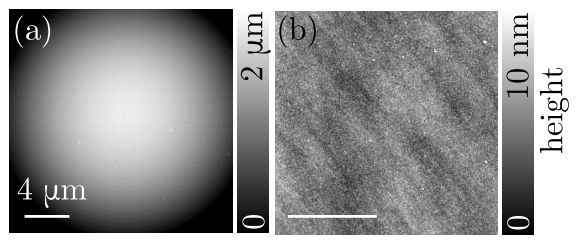

(c)
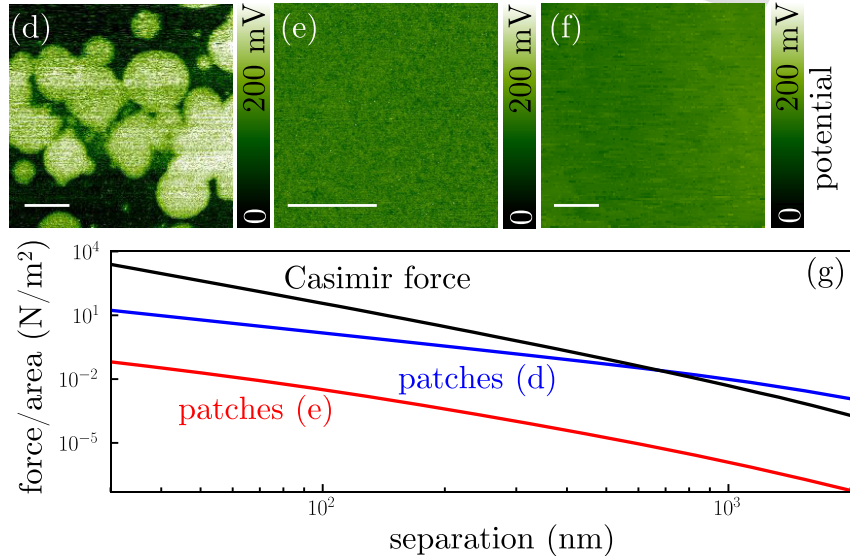

FIG. 1. Surface potential of gold-coated spherical probes scanned with Kelvin probe force microscopy (KPFM). (a) Topography and (d) KPFM voltage for a spherical probe without an ionblocking layer showing spatial voltage variation. (b), (e) Similar data for a plate with $\mathrm{a} \mathrm{SiO}_{2}$ blocking layer with little spatial variation. (c) The imaging setup. (f) Surface potential of a spherical probe with a $\mathrm{TiO}_{2}$ blocking layer. (g) The patch potential forces from surfaces (d), (e) relative to the Casimir force, in the planar approximation.

(Mikromasch HQ:NSC35/Pt) in an atomic force microscope (Asylum Research, Cypher). The spherical probe is held at $11^{\circ}$ relative to the horizontal so that the region of the sphere that comes closest to the plate is the region that is imaged [Fig. 1(c)].

Measurements of the surface potential on gold-coated spheres show that patch potentials are affected by the substrate on which the 100-nm-thick gold film is deposited [Figs. 1(b)1(f)]. More patches are observed on a glass sphere (Trelleborg SI-100) than on a Si plate when a 3-nm Cr layer is used for adhesion.

We suspect that the patches result from mobile impurity ions in the glass, which can migrate through the thin gold layer, but we also note that similar patches have been attributed to trapped charges on the substrate [35]. When an ion-blocking layer is deposited between the glass and the gold film, the electrostatic patches are significantly smaller. For example, both a 50-nm $\mathrm{SiO}_{2}$ ion-blocking layer with a 3-nm $\mathrm{Cr}$ sticking layer and a $10-\mathrm{nm} \mathrm{TiO}_{2}$ ion-blocking layer with a 3-nm Ti sticking layer reduced the spatial extent and voltage contrast of the patches [Figs. 1(e) and 1(f)]. Figure 1(g) compares the patch potential forces from the surfaces depicted in Figs. 1(d) and 1(e) to the Casimir force. While the force calculated from Fig. 1(e) is consistent with the previous bound on patch potentials, the force from Fig. 1(d) is significantly larger and even exceeds the magnitude of the Casimir force at separations greater than $700 \mathrm{~nm}$.

KPFM images of the sphere and the plate from both before and after each measurement are used to determine the range

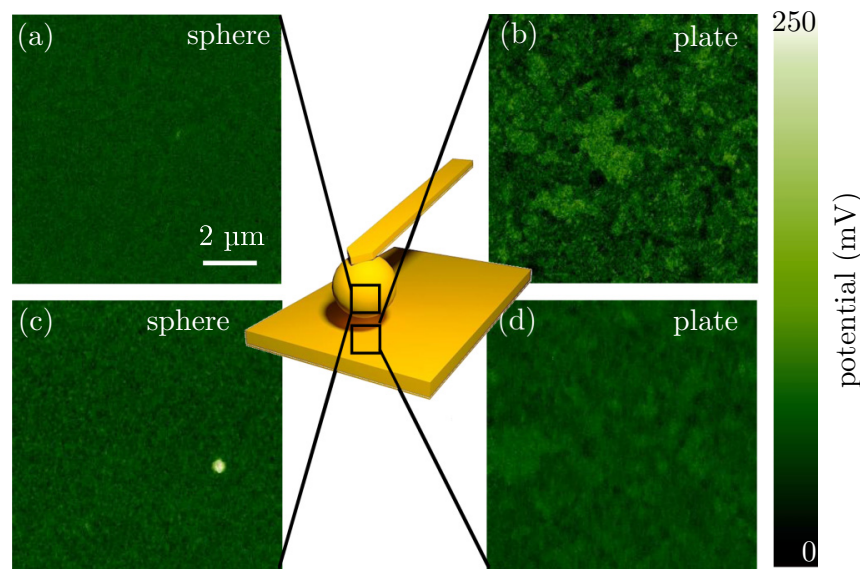

FIG. 2. KPFM images of the gold-coated spheres (left column) and plates (right column) used for two different measurement sets (a), (b) and (c), (d).

of possible forces due to patch potentials. (Figure 2 shows KPFM scans before force measurements.) No significant difference in the calculated electrostatic patch force is observed when using the KPFM images acquired before or after the force measurements. Different cantilevers are used for the different scans, so we report the frequencies used with a representative cantilever. For example, to acquire a KPFM image an applied $\mathrm{AC}$ voltage of $5 \mathrm{~V}$ at a driving frequency of $316.35 \mathrm{kHz}$, a topographical loop at $158.68 \mathrm{kHz}$ (a few hertz larger than the first eigenmode), and a detection frequency of $157.68 \mathrm{kHz}$ were used. The $\mathrm{TiO}_{2}$ blocking layer is present on the spheres, and both silicon plates are coated with a 3-nm Ti adhesion layer and $100 \mathrm{~nm}$ of gold. The two spherical probes are fabricated simultaneously, and the same plate is used for both measurements, although the location differs.

\section{FORCE MEASUREMENTS}

The spatial derivative of the Casimir force $F_{C}^{\prime}$ is measured between a sphere and a plate by oscillating the plate along the $z$ axis and observing the response of the probe [27-29,36]. The plate moves towards and away from the sphere in discrete steps. The measurements are performed in air at $303.15 \pm$ $0.05 \mathrm{~K}$ and $30 \pm 2 \%$ relative humidity over the course of 55 or $36 \mathrm{~h}$, depending on the measurement run. The spring constant $k$ of the first probe is $0.043 \mathrm{~N} / \mathrm{m}$ and the spring constant $k$ of the second probe is $0.25 \mathrm{~N} / \mathrm{m}$, and the radii of the spheres are 24.6 and $35.8 \mu \mathrm{m}$, respectively (mounted on Bruker MLCT-O10). Using two cantilevers with different $k$ helps to ensure that there are no small, unanticipated effects (inversely) proportional to $k$.

The sensitivity and separation between the sphere and the probe are determined via an electrostatic force calibration $[28,36]$. Two electrostatic signals are driven, one proportional to the force $[27,28]$ and one proportional to the force gradient [36]. The former has a much higher signal-to-noise ratio, so it is used to track drift in $k$ and $d$, while the latter has a better accuracy, so it is used for absolute calibration. The voltages which, when applied to the probe, minimize the electrostatic force or force gradient are also recorded at each separation. 
At separations of less than $100 \mathrm{~nm}$, the voltage driving the electrostatic force-gradient signal is turned off to prevent the probe and the plate from sticking together. Both the plate oscillation and the voltage are applied at frequencies below the cantilever resonance: 211 and $77 \mathrm{~Hz}$, respectively.

\section{CALCULATION OF PATCH POTENTIAL FORCES}

Both the residual electrostatic contribution to the measurement and the minimizing voltages are calculated from the KPFM scans, so that they can be compared to the measured values. Speake and Trenkel (ST) [19] devised a method to calculate the force from patch potentials on parallel plates by modeling them as a dipole layer on each surface just above the surface of a perfect conductor. ST wrote these formulas for parallel plates in terms of the surface potential $k$-space autocorrelation function, which can be calculated from KPFM scans [26]. To use the ST formulas for curved surfaces, they are transformed from $k$-space into real space in order to facilitate their incorporation into the proximity force approximation. In real space, the force and the force gradient from patch potentials are written as

$$
F^{(n)}=-\frac{\varepsilon_{0}(n+1) !}{2} \iint \sum_{i, j=0,1} \frac{(-1)^{\alpha+n} V_{i} \tilde{V}_{i j}^{(n)}(h)}{h^{2+n}} d^{2} \vec{x},
$$

where $n=0$ or 1 denotes force or gradient, $i, j=0$ or 1 denotes plate or sphere, $\alpha=|i-j|, V_{i}$ is the potential on one surface, $h(x, y)$ is the separation between the curved surfaces at lateral position $(x, y)$, and $\tilde{V}_{i j}^{(n)}$ is the effective potential due to charges from surface $j$ at surface $i$, defined as the convolution of $V_{j}$ with one of the radially symmetric functions $g_{i j}^{(n)}$ which are in turn defined as

$$
g_{i j}^{(n)}(h, r)=\frac{(-h)^{2+n}}{2 \pi(n+1) !} \int_{0}^{\infty} \frac{d^{n} f_{i j}}{d h^{n}} J_{0}(k r) d k,
$$

where both of the functions $f_{i i}=k^{3} / \sinh (k h)^{2}$ and $f_{i \neq j}=$ $k^{3} \cosh (k h) / \sinh (k h)^{2}$ originate from the version of the ST equation found in Ref. [21], and $r$ is the radial coordinate. The uncertainty in the orientation of the sphere is propagated to determine the uncertainty in the force and the minimizing voltage [37].

One of the distinguishing features of patch potentials in sphere-plate measurements is how the expected force and force-gradient minimizing voltages change with separation $[20,38]$. The change in the minimizing voltage with separation originates from how different regions of the surfaces contribute to the total electrostatic force in different proportions depending on the separation between the surfaces. Because the minimizing voltages do not change in the parallel plate geometry, a direct application of the proximity force approximation to the $k$-space calculation predicts no changes [21].

Behunin et al. [22] present a method to compute the patch potential force exactly for a sphere and a plate, but it is computationally intensive and limited to micron-scale patches. In real space, the force and force-gradient minimizing voltages can be calculated from Eq. (1) in the proximity force approximation from images of the potential at the resolution acquired by KPFM. Measurements of the minimizing voltage validate the computation of the patch potential force.

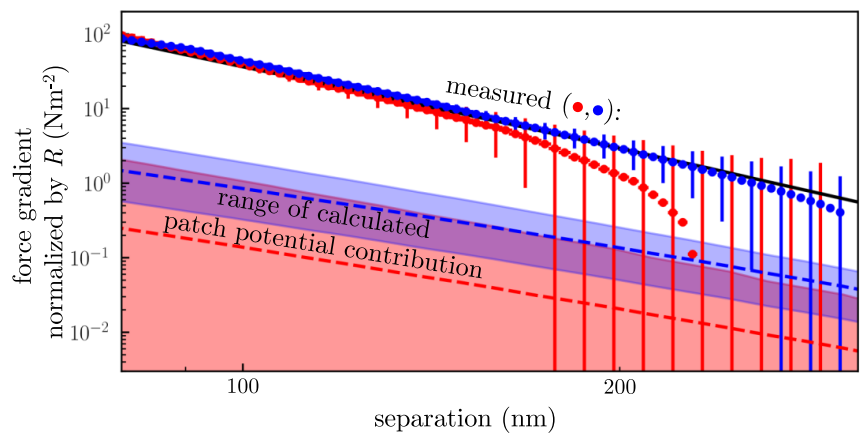

FIG. 3. The force measured between the first (blue) and the second (red) sphere and plate sets compared to the calculated and measured Casimir force (solid black line and solid circles, respectively). The electrostatic patch potential force is determined to be within the red or blue shaded range, which is calculated from $10^{4}$ possible sphere orientations, and the dashed lines indicate the median for each sphere.

The uncertainty in the electrostatic interaction is determined by calculating the patch potential force gradient for many different orientations of the sphere. First, a $5 \times 5$ grid of points $(5 \times 5 \mu \mathrm{m})$ is placed on each KPFM scan of a sphere and plate set. The regions of the two surfaces outside of the KPFM scan region are assumed to be at an equipotential. The electrostatic force is calculated while modeling the sphereplate system so that one of the points in the grid on the sphere is directly above one of the grid points on the plate. The calculation of both the force (gradient) minimizing voltage and the force (gradient) at the minimizing voltage is repeated for separations ranging from $70 \mathrm{~nm}$ to $1 \mu \mathrm{m}$, for each sphereplate pair of points, and for four relative orientations of the two surfaces.

\section{COMPARISON OF FORCES}

The calculated patch potential force gradients are compared directly to the measured force and the force calculated from measured dielectric data [36,39] (Fig. 3). The calculated electrostatic force gradients from all the sphere orientations are more than an order of magnitude less than the measured force gradient, at separations up to 240 and $160 \mathrm{~nm}$ for the first and second data sets, respectively. Beyond those separations, the hydrodynamic force is the limiting source of error.

The force-gradient minimizing voltage is consistent with the predictions of the electrostatic calculation for both sphereplate sets, which is an additional indicator of the validity of the ST calculation of the electrostatic patch force. The force minimizing voltage is consistent with only the first set (see Fig. 4 and the discussion below). All the minimizing voltages are plotted relative to the value of each at the smallest achieved separation. For reference, the relative difference between the force minimizing voltage and the force-gradient minimizing voltage is 3.0 and $2.5 \mathrm{mV}$ at a $200-\mathrm{nm}$ separation, for each sphere respectively.

There are two possible reasons why the force minimizing voltage for the second sphere-plate set is inconsistent with the ST calculation. First, it is possible that a large patch on the sphere outside the scanned region leads to a force 

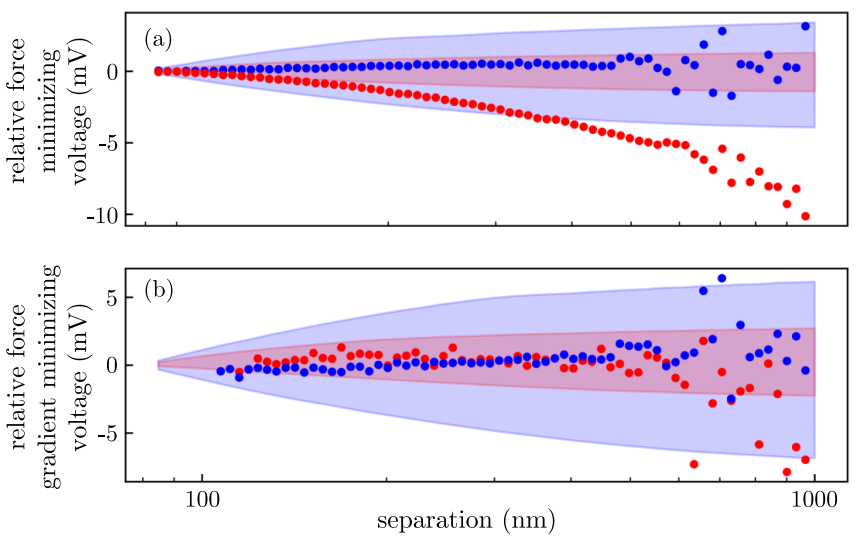

FIG. 4. The relative (a) force and (b) force-gradient minimizing voltages as a function of separation between the sphere and the plate. The dots show measured values and the shaded regions show the range of possible values calculated from the KPFM images for the first (blue) sphere and second (red) sphere.

minimizing voltage that is greater than expected. The small high-contrast patch on the sphere suggests that a large unseen patch is a possibility [see Fig. 2(c)]. Second, AC coupling could have led to the appearance of an artificial voltage shift, as is common in KPFM [40].

\section{CONCLUSIONS}

By determining the electrostatic patch potential force using measured KPFM images, we confirm that the measured force is the Casimir force and not due to spurious electrostatic interactions. However, KPFM images of gold-coated glass spheres without an ion-blocking layer reveal the presence of large patches in other samples, which could mimic the Casimir force if not taken into account. Moreover, these measurements show that KPFM scans could be used to validate cleaning procedures used to mitigate the effect of patch potentials from similar Casimir force measurements [41]. An ion-blocking layer is an effective way to eliminate these potentials from gold-coated glass spheres and may be an effective way to mitigate patch potentials in other systems as well. For example, Wang et al. [35] discuss an experiment in which two different materials, gold and silicon, are coated with the same metal layer to eliminate electrostatic and Casimir force contrast between them, but they find that the electronic contrast is not eliminated by the gold coating until it is several hundred nanometers thick and annealed. However, even with the blocking layer, the estimated contribution of the patch force to the total Casimir force measurements in some possible sphere-plate configurations is larger than the largest estimates from previous measurements on gold plates [25,26]. For example, at all separations examined here, there exist a few configurations that result in a patch force gradient $>2 \%$ of the Casimir force gradient.

These experiments also demonstrate the possible influence of electrostatic inhomogeneities on microelectromechanical systems and show the need to directly test the ST formulation of patch potential forces in a setting where they are the dominant force in order to validate its use in characterizing uncertainties. The development of KPFM in liquids [42] may enable a similar study of patch potentials in liquid environments, where electrostatics are different [43], but in which the Casimir force exhibits exotic behavior [44,45]. KPFM with atomic resolution would help to identify the underlying cause of the patch potentials, which in turn may help to eliminate them $[18,21]$. Finally, scanning probe techniques that measure other surface inhomogeneities will allow the quantification of other patch forces, such as the force from magnetic domains, which may be present in materials with $\mu \neq 1$ that are of interest in the search for repulsive and thermal Casimir forces [46].

\section{ACKNOWLEDGMENT}

We acknowledge funding from DARPA YFA, Grant No. D18AP00060.
[1] H. B. G. Casimir, Proc. K. Ned. Akad. Wet. 51, 793 (1948).

[2] M. Sparnaay, Physica 24, 751 (1958).

[3] P. H. G. M. van Blokland and J. T. G. Overbeek, J. Chem. Soc., Farad. Trans. 1 74, 2637 (1978)

[4] S. K. Lamoreaux, Phys. Rev. Lett. 78, 5 (1997).

[5] U. Mohideen and A. Roy, Phys. Rev. Lett. 81, 4549 (1998).

[6] W. J. Kim, A. O. Sushkov, D. A. R. Dalvit, and S. K. Lamoreaux, Phys. Rev. Lett. 103, 060401 (2009).

[7] A. O. Sushkov, W. J. Kim, D. A. R. Dalvit, and S. K. Lamoreaux, Nat. Phys. 7, 230 (2011).

[8] J. Laurent, H. Sellier, A. Mosset, S. Huant, and J. Chevrier, Phys. Rev. B 85, 035426 (2012).

[9] J. Zou, Z. Marcet, A. W. Rodriguez, M. T. H. Reid, A. P. McCauley, I. I. Kravchenko, T. Lu, Y. Bao, S. G. Johnson, and H. B. Chan, Nat. Commun. 4, 1845 (2013).

[10] Z. Babamahdi, V. B. Svetovoy, M. Enache, M. Stöhr, and G. Palasantzas, Phys. Rev. B 100, 245422 (2019).

[11] J. M. McGuirk, D. M. Harber, J. M. Obrecht, and E. A. Cornell, Phys. Rev. A 69, 062905 (2004).
[12] M. Brownnutt, M. Kumph, P. Rabl, and R. Blatt, Rev. Mod. Phys. 87, 1419 (2015).

[13] R. W. Andrews, A. P. Reed, K. Cicak, J. D. Teufel, and K. W. Lehnert, Nat. Commun. 6, 10021 (2015).

[14] Y. Pu, D. D. Neufeld, and F. B. Dunning, Phys. Rev. A 81, 042904 (2010).

[15] C. W. F. Everitt, D. B. DeBra, B. W. Parkinson, J. P. Turneaure, J. W. Conklin, M. I. Heifetz, G. M. Keiser, A. S. Silbergleit, T. Holmes, J. Kolodziejczak et al., Phys. Rev. Lett. 106, 221101 (2011).

[16] F. Antonucci, A. Cavalleri, R. Dolesi, M. Hueller, D. Nicolodi, H. B. Tu, S. Vitale, and W. J. Weber, Phys. Rev. Lett. 108, 181101 (2012).

[17] N. D. Lang and W. Kohn, Phys. Rev. B 3, 1215 (1971).

[18] P. Ruffieux, K. Aït-Mansour, A. Bendounan, R. Fasel, L. Patthey, P. Gröning, and O. Gröning, Phys. Rev. Lett. 102, 086807 (2009).

[19] C. C. Speake and C. Trenkel, Phys. Rev. Lett. 90, 160403 (2003). 
[20] W. J. Kim, A. O. Sushkov, D. A. R. Dalvit, and S. K. Lamoreaux, Phys. Rev. A 81, 022505 (2010).

[21] R. O. Behunin, F. Intravaia, D. A. R. Dalvit, P. A. Maia Neto, and S. Reynaud, Phys. Rev. A 85, 012504 (2012).

[22] R. O. Behunin, Y. Zeng, D. A. R. Dalvit, and S. Reynaud, Phys. Rev. A 86, 052509 (2012).

[23] C. D. Fosco, F. C. Lombardo, and F. D. Mazzitelli, Phys. Rev. A 88, 062501 (2013).

[24] D. Garcia-Sanchez, F. Fong, H. Bhaskaran, S. Lamoreaux, and H. Tang, Phys. Rev. Lett. 109, 027202 (2012).

[25] R. O. Behunin, D. A. R. Dalvit, R. S. Decca, C. Genet, I. W. Jung, A. Lambrecht, A. Liscio, D. Lopez, S. Reynaud, G. Schnoering, G. Voisin, and Y. Zeng, Phys. Rev. A 90, 062115 (2014).

[26] J. L. Garrett, D. A. T. Somers, and J. N. Munday, J. Phys.: Condens. Matter 27, 214012 (2015).

[27] S. de Man, K. Heeck, R. Wijngaarden, and D. Iannuzzi, Phys. Rev. Lett. 103, 040402 (2009).

[28] S. de Man, K. Heeck, and D. Iannuzzi, Phys. Rev. A 82, 062512 (2010).

[29] J. L. Garrett, D. A. T. Somers, and J. N. Munday, Phys. Rev. Lett. 120, 040401 (2018).

[30] Y. Sugawara, L. Kou, Z. Ma, T. Kamijo, Y. Naitoh, and Y. Jun Li, Appl. Phys. Lett. 100, 223104 (2012).

[31] J. M. Obrecht, R. J. Wild, and E. A. Cornell, Phys. Rev. A 75, 062903 (2007).

[32] C. P. Blakemore, A. D. Rider, S. Roy, Q. Wang, A. Kawasaki, and G. Gratta, Phys. Rev. A 99, 023816 (2019).

[33] J. L. Garrett and J. N. Munday, Nanotechnology 27, 245705 (2016).
[34] J. L. Garrett, L. J. Krayer, K. J. Palm, and J. N. Munday, Appl. Phys. Lett. 111, 043105 (2017).

[35] J. Wang, S. Guan, K. Chen, W. Wu, Z. Tian, P. Luo, A. Jin, S. Yang, C. Shao, and J. Luo, Phys. Rev. D 94, 122005 (2016).

[36] J. L. Garrett, D. A. T. Somers, K. Sendgikoski, and J. N. Munday, Phys. Rev. A 100, 022508 (2019).

[37] R. I. P. Sedmik, A. Almasi, and D. Iannuzzi, Phys. Rev. B 88, 165429 (2013).

[38] S. de Man, K. Heeck, and D. Iannuzzi, Phys. Rev. A 79, 024102 (2009); W. J. Kim, M. Brown-Hayes, D. A. R. Dalvit, J. H. Brownell, and R. Onofrio, ibid. 78, 020101(R) (2008).

[39] A. Lambrecht and S. Reynaud, Eur. Phys. J. D 8, 309 (2000).

[40] S. Barbet, M. Popoff, H. Diesinger, D. Deresmes, D. Théron, and T. Mélin, J. Appl. Phys. 115, 144313 (2014).

[41] J. Xu, G. L. Klimchitskaya, V. M. Mostepanenko, and U. Mohideen, Phys. Rev. A 97, 032501 (2018).

[42] L. Collins, J. I. Kilpatrick, S. V. Kalinin, and B. J. Rodriguez, Rep. Prog. Phys. 81, 086101 (2018).

[43] J. N. Munday, F. Capasso, V. A. Parsegian, and S. M. Bezrukov, Phys. Rev. A 78, 032109 (2008).

[44] J. N. Munday, F. Capasso, and V. A. Parsegian, Nature (London) 457, 170 (2009).

[45] R. Zhao, L. Li, S. Yang, W. Bao, Y. Xia, P. Ashby, Y. Wang, and X. Zhang, Science 364, 984 (2019).

[46] G. Bimonte, D. López, and R. S. Decca, Phys. Rev. B 93, 184434 (2016).

Correction: A relevant citation was missing and has been added as the second part of Ref. [38]. 